\title{
LA CONSTRUCCIÓN HISTÓRICA DE LA COREA DEL SUR CONTEMPORÁNEA A TRAVÉS DEL CINE NEGRO: \\ EL CASO DE NAMELESS GANGSTER (2013) Y GANGNAM BLUES (2015)
}

\author{
Luis Miguel Machín Martín \\ Universidad de La Laguna
}

\section{RESUMEN}

Corea del Sur afrontó su transición democrática en la segunda mitad de los años 80 después de décadas de dictadura militar. Su actual situación política y económica está muy influida por esa etapa, que se caracterizaba por la corrupción institucional y el auge del crimen organizado. En el cine surcoreano, las mejores representaciones de esa época se han realizado bajo el paraguas del género negro, que envuelve esas problemáticas en un disfraz de cine criminal que las hace más digeribles comercialmente. ése es el caso de Nameless Gangster y Gangnam Blues, dos películas ambientadas en los ańos 80 y 70 , respectivamente, que tratan, en clave noir, la corrupción política y el crecimiento de las organizaciones mafiosas en esa época en el país. Este artículo analiza cómo estas dos películas han representado esas dos décadas fundamentales para la historia reciente surcoreana, centrándose, sobre todo, en su exposición de la pobreza, la desigualdad y la corrupción.

Palabras Clave: Corea del Sur, cine surcoreano, cine negro, corrupción, mafia.

\section{THE HISTORICAL MAKING OF THE CONTEMPORARY SOUTH KOREA THROUGH THE FILM NOIR: THE CASES OF NAMELESS GANGSTER (2013) AND GANGNAM BLUES (2015)}

\section{Abstract}

South Korea faced its democratic transition in the second half of the 80 s after decades of military dictatorship. Its current political and economic situation is strongly influenced by this stage, which was characterized by institutional corruption and the rise of organized crime. The best representations of that era in the South Korean cinema have been made in the film noir, which has been capable of make these matters more commercially approachable. That is the case of Nameless Gangster and Gangnam Blues, two films set in the 80s and 70s, respectively, that deal with the political corruption and the growth of mafia organizations at that time in the country. This paper analyzes how these two films have represented those two decades that are fundamental for South Korean recent history, focusing fundamentally on their exposure of poverty, inequality and corruption.

Keywords: South Korea, South Korean cinema, film noir, corruption, organized crime. 


\section{INTRODUCCIÓN}

Desde que a mediados de los años 80 comenzara su transición a la democracia después de décadas de dictadura militar, Corea del Sur realizó un rápido desarrollo económico basado en el liberalismo ${ }^{1}$-que se aplicó con especial vehemencia en el sector cinematográfico ${ }^{2}-$ y en una industrialización tardía caracterizada por la diversificación ${ }^{3}$, que le permitieron convertirse en una de las potencias emergentes en Asia. En ese proceso de transición Corea del Sur atravesó dos fases fundamentales desde el fin de la dictadura: la primera, que abarca de junio de 1987 a 1992, que se considera la etapa de la transición democrática (aunque algunos autores ven su inicio en 1985); la segunda, a partir de ese 1992, momento en que ya se puede hablar de consolidación de la democracia surcoreana ${ }^{4}$.

Sin embargo, cuestiones como la corrupción política e institucional y el auge del crimen organizado ${ }^{5}$ provocaron que la transición democrática y los primeros lustros de democracia fueran problemáticos y complicaran la estabilización de la democracia recién instituida. La sociedad tuvo que adaptarse a una transformación casi instantánea, pasando de una dictadura militar a una democracia fuertemente influida por las instituciones y actores de la época dictatorial, lo que provocó un enfrentamiento entre diferentes actores civiles, algunos de los cuales exigían la instauración de una democracia plena con objetivos ambiciosos (como la igualdad socioeconómica o la reunificación de Corea) mientras otros proponían una definición de democracia más estrecha y controlada ${ }^{6}$. Esa rápida y forzada adaptación tuvo consecuencias negativas en la población surcoreana, que tuvo que afrontar una serie de amenazas como «el autoritarismo político y patriarcal, prácticas despóticas y monopolísticas de los chaebol ${ }^{7}$, la explotación y exclusión laboral o la desatención de los derechos básicos de la sociedad de bienestar» ${ }^{8}$.

A pesar de haber sufrido algún duro altibajo como el que afectó al país a finales de los años 90 con la crisis asiática, que requirió que el Fondo Monetario

${ }^{1}$ Demetriades, E.O. y Luintel, K.B. (2000): «Financial restraints in the South Korean miracle», Journal of Development Economics, vol. 64, núm. 1, pp. 461-462.

${ }_{2}$ Jin, Dal Yong (2006): «Cultural politics in Korea's contemporary films under neoliberal globalization», Media, Culture \& Society, vol. 28, núm. 1, p. 5.

3 Amsden, Alice H. (1992): Asia's Next Giant: South Korea and Late Industrialization, p. 7. Oxford, Oxford University Press.

${ }^{4}$ LeE, Sangmook (2007): «Democratic Transition and the Consolidation of Democracy in South Korea», Taiwan Journal of Democracy, vol. 3, núm. 1, p. 101.

${ }^{5}$ LeE, Seungmug (2006): «Organized Crime in South Korea», Trends in Organized Crime, vol. 9, núm. 3, pp. 65-66.

${ }^{6}$ KIm, Sunhyuk (1997): «State and Civil Society in South Korea’s Democratic Consolidation: Is the Battle Really over?», Asian Survey, vol. 37, núm. 12, p. 1137.

7 Los chaebol son grandes conglomerados industriales de tradición familiar de Corea del Sur, que abarcan un numeroso conjunto de actividades económicas. Hyundai, LG o Samsung son ejemplos de chaebol.

${ }^{8}$ Chang, Kyung-sup (1999): «Compressed modernity and its discontents: South Korean society in transition», Economy and Society, vol. 28, núm. 1, p. 30. 
Internacional interviniera para apoyar económicamente al país ${ }^{9}$, Corea del Sur continuó su camino hasta convertirse, en la actualidad, en una de las potencias económicas de Asia.

Su cine, por otro lado, emergió rápidamente a finales del siglo $\mathrm{xx}$, cuando los chaebol identificaron la industria audiovisual como un sector económicamente estratégico, dando como resultado una gran inversión en dicha industria a partir de mediados de los ańos $90^{10}$. Esa inversión proporcionó al mundo audiovisual surcoreano un impulso que ha mantenido a su cinematografía en constante expansión desde entonces. Sin embargo, con la mencionada crisis y con el correspondiente rescate del FMI, se impusieron una serie de restricciones económicas a las empresas e instituciones del país, entre ellas que los principales chaebol renunciaran a su participación en la industria audiovisual, dejando un vacío para que otros chaebol de segundo nivel aprovecharan la circunstancia y se convirtieran en los principales referentes de la producción y distribución cinematográfica en Corea del Sur ${ }^{11}$.

Así, en el último tramo de la década de los 90 comenzaron a producirse los primeros grandes éxitos en taquilla como Shiri (Kang Je-gyu, 1999), que abrió la puerta a otros éxitos comerciales posteriores como Joint Securiy Area (Park Chanwook, 2000), y que fueron el germen del renacimiento del cine surcoreano ${ }^{12}$. A partir de ese momento se produce un crecimiento tal en el cine y en la cultura popular del país que se crea la etiqueta de hallyu (literalmente ola coreana en coreano) para referirse a este movimiento cultural en constante ebullición ${ }^{13}$. La cultura popular coreana se ha expandido desde entonces por toda Asia y parte del resto del mundo de forma rápida e imparable, especialmente en lo que respecta a su música pop $(K-P o p)^{14}$, a su cine, a su televisión y, en menor medida, a su literatura.

Esa ola coreana está caracterizada en su apartado cinematográfico, entre otras cosas, por:

${ }^{9}$ CHo, Younghan (2008): «The national crisis and de/reconstructing nationalism in South Korea during the IMF intervention», Inter-Asia Cultural Studies, vol. 9, núm. 1, p. 82.

10 Ryoo, Wongjae (2008): «The political economy of the global mediascape: the case of the South Korean film industry», Media, Culture \& Society, vol. 30, núm. 6, p. 875.

${ }^{11}$ SHIm, Doobo (2006): «Hybridity and the rise of Korean popular culture in Asia», Media, Culture \& Society, vol. 28, núm. 1, p. 33.

12 Kim, Sung Kyung (2006): “'Renaissance of Korean National Cinema' as a Terrain of Negotiation and Contention between the Global and the Local: Analysing two Korean Blockbusters, Shiri (1999) and JSA (2000)», The Essex Graduate Journal of Sociology, vol. 15, núm. 1, pp. 83-97.

13 YANG, Jonghoe (2012): "The Korean wave (Hallyu) in East Asia: A comparison of Chinese, Japanese, and Taiwanese audiences who watch Korean TV dramas», Development and Society, vol. 41, núm. 1, p. 104.

${ }^{14}$ Kim, E.M. y Ryoo, J. (2007): «South Korean Culture Goes Global: K-Pop and the Korean Wave», Korean Social Science Journal, vol. 34, núm. 1, pp. 117-152. 
- Un gran aumento del número de producciones cinematográficas anuales: mientras que en 1995 se estrenaron 62 películas surcoreanas, en 2010 el número ya se había disparado a 142, y en 2016 la cifra ya estaba en $339^{15}$.

- La identificación del público con el cine nacional, que hace que sus películas obtengan un gran share año tras año en la taquilla anual del país. Así, desde el 2001 el cine coreano ha conseguido, casi sin excepciones, al menos un $50 \%$ de la taquilla total año tras año, según datos del Korean Film Council (KOFIC).

- La creación de un star system de intérpretes, que aseguran con su presencia, prácticamente, el éxito de la película en que participan ${ }^{16}$.

- Y, finalmente, la aparición de una generación de directores capaz de combinar toques artísticos y autorales con una gran capacidad comercial, generación que ha ganado premios en la mayor parte de los mejores festivales de cine del mundo ${ }^{17}$ y que ha impulsado a la marca del cine coreano, que ha sido calificado, también, de well-made (bien hecho), en referencia a su lograda factura técnica ${ }^{18}$.

Además, se da la circunstancia de que una buena parte de esos directores han dirigido cine negro o criminal, lo que ha supuesto una revitalización del género. Así, cineastas como Park Chan-wook, Bong Joon-ho, Kim Jee-woon o Na Hong-jin dirigen películas a menudo sobre asesinos en serie, sobre complejas investigaciones policiales, sobre la mafia y los bajos fondos, sobre secuestros o, en general, thrillers criminales.

\section{CINE DE GÁNGSTERES EN COREA DEL SUR: ENTRE LA CRÍTICA Y LA REVISIÓN HISTÓRICA}

El cine de gángsterEs se ha convertido en uno de los géneros más rentables dentro de la ola coreana, alimentando temáticamente no sólo al cine negro sino a otros géneros como el drama o la comedia, que incluyen temáticas relacionadas con el crimen organizado ${ }^{19}$. Dramas de diferente tratamiento y tono como Bad Guy (Kim Ki-duk, 2001), Friend (Kwak Kyung-taek, 2001) -la primera, una obra autoral; la

\section{Corea del Sur.}

15 Datos extraídos de la web del KOFIC, organismo oficial de promoción del cine de

${ }^{16}$ Cueto, Roberto (2004): «Un nuevo cine para una nueva realidad (o las películas que los coreanos quieren ver)", en Elena, A. (ed.), Seúl Express 97-04, p. 28, Madrid, T\&B Editores.

${ }_{17}$ Park Chan-wook ha ganado premios como el Gran Premio y el Premio del Jurado de Cannes; Bong Joon-ho ha obtenido diversos premios en el Festival de San Sebastián y Mar del Plata; Hong Sang-soo ha sido premiado en Cannes, Rotterdam, Locarno y San Sebastián; Kim Ki-duk ha sido premiado varias veces en Venecia; etc.

${ }_{18}$ CHoI, Jinhee (2010): The South Korean Film Rennaissance: Local Hitmakers, Global Provocateurs, Middletown, Connecticut, Wesleyan University Press, p. 144.

19 Ibidem, p. 60. 
segunda, un gran blockbuster-o My Wife is a Gangster (Cho Jin-gyu, 2001), comedia romántica de trasfondo criminal, son ejemplos de éxito de esta temática en el inicio de la ola coreana, éxito que va a propiciar que las películas de gángsterEs continúen haciéndose desde ese momento de una forma más o menos calculada y casi seriada.

En esta etapa temprana de la ola coreana, a comienzos del siglo XxI, el cine de tema mafioso ofrece una visión exterior del funcionamiento del crimen organizado, sin mostrar sus mecanismos internos y sin asociar su existencia a una coyuntura social, política o histórica específica ${ }^{20}$. Esa visión superficial de la mafia poco a poco cambia y va apareciendo un numeroso conjunto de obras que contarán historias desde el interior de las bandas de gángsterEs.

Si bien hay antecedentes de esta representación interior del crimen organizado en los albores del hallyu, como Green Fish (Lee Chang-dong, 1997), no será hasta 2005 cuando las tramas criminales comenzarán a contarse regularmente desde las entrañas de las bandas mafiosas. Es en ese momento cuando surge $A$ Bittersweet Life (Kim Jee-woon, 2005), un thriller que narra las peripecias de un habilidoso matón que tendrá que enfrentarse a su propia banda cuando su jefe descubra que su prometida ha tenido un affaire con él. A partir de esta influyente obra se da paso a toda una serie de películas coreanas de solitarios antihéroes que pertenecen o están enfrentados a una banda de criminales.

Poco a poco el cine negro surcoreano empezaba a acercarse a temáticas más sociales -sin que eso significara dejar de lado la voluntad de hacer cine de entretenimiento- o con un trasfondo más realista, más histórico. Así, se comenzaba a hacer común la crítica a ciertas instituciones públicas del país. Por ejemplo, la policía no deja de ser criticada, siendo representada como un estamento incompetente y corrupto ${ }^{21}$. Películas como Memories of Murder (Bong Joon-ho, 2003) o The Chaser (Na Hong-jin, 2008), ambas grandes representantes del noir de la ola coreana, muestran como a unos ineptos a los detectives y policías que participan en sus tramas e investigaciones.

Este tipo de críticas abrieron la puerta a un cierto revisionismo del pasado próximo del país, ofreciendo desde entonces una visión desencantada en el cine del camino seguido por la nación desde la transición democrática. De este modo empiezan a realizarse películas basadas en hechos reales como la mencionada Memories of Murder ${ }^{22}$ y aparecen otras obras que, sin partir de ningún hecho concreto, sí que

${ }^{20}$ Machín Martín, Luis Miguel (2013): «La mafia en el cine criminal coreano contemporáneo: características e influencias», en Herrero, J., Sánchez Pita, F., Ardévol, A. y ToleDANO, S. (coords.), La sociedad ruido: entre el dato y el grito, p. 8, La Laguna, Sociedad Latina de Comunicación Social.

${ }^{21}$ Lee, Hyun-jung (2015): «Portraying police in Korean cinema». Korean Herald. Online: http://www.koreaherald.com/view.php?ud=20151204000938. [Consultado el 03/02/2019].

${ }^{22}$ Memories of Murder cuenta la historia real del caso de Hwaseong, una serie de asesinatos ocurridos entre 1986 y 1991 en la región homónima de Corea del Sur. Esta película ofrecía, al margen de las intrigas propias del cine de detectives, una radiografía del estado del país en la época de la transición hacia la democracia. 
hablan de una coyuntura histórica específica. Ese es el caso de Nameless Gangster (Yun Jong-bin, 2012) y Gangnam Blues (Ha Yu, 2015).

Nameless Gangster y Gangnam Blues están ambientadas en los años 80 y 70, respectivamente, y narran, en ambos casos, cómo la corrupción política está estrechamente relacionada con el ascenso del crimen organizado y, a su vez, ambos factores están unidos a la convulsa época que fue la transición democrática del país. Realizadas más de 20 años después del fin de la dictadura en Corea del Sur, estas dos películas aparecen en un contexto de relativa madurez institucional del Estado, disponiendo de una perspectiva suficientemente amplia para poder observar los aciertos y errores cometidos en el pasado más próximo de la nación. Ahí es donde el género negro y, más concretamente, el cine de gángsteres cumplen una función de reflexión histórica.

El género negro, desde su nacimiento, ha servido de canalizador de desencanto sobre el presente y el pasado del lugar donde es concebido. Así ocurrió con el surgimiento del cine negro original, el del periodo clásico de Hollywood, que, tal y como expone Paul Schrader en su famoso texto Notes on film noir, coincide con un momento histórico de "cinismo, pesimismo y oscuridad que había arrastrado al cine norteamericano" ${ }^{23}$. Y así ha ocurrido en Corea del Sur en el contexto de la ola coreana, movimiento que ha coincidido con el avance del neoliberalismo en el país, con unas duras condiciones laborales para una buena proporción de la población, una globalización que llegó rápidamente, tras décadas de autoritarismo, pasando de un duro hermetismo a un enorme aperturismo en pocos años ${ }^{24}$, y con los últimos coletazos de la influencia del régimen militar durante los ańos $90^{25}$.

Así, el cine negro se convierte en una herramienta de revisión del pasado próximo del país. Este cine, utilizando la crónica negra reciente del país (asesinatos, secuestros y diferentes temáticas criminales), ha mezclado elementos clásicos del género y una revisión histórica de lo que ha sido Corea del Sur, de lo que es y de lo que quiere ser. Es, por tanto, un género que en este país asiático se ha usado como narrador de su evolución social y que funciona como uno de los sectores artísticos menos frívolos dentro de la ola coreana.

${ }^{23}$ Schrader, Paul (1972): «Notes on film noir». Film Comment, vol. 8, núm. 1, p. 53.

${ }^{24}$ Los Juegos Olímpicos de Seúl 88 fueron el primer gran acto de aperturismo de un país que llevaba décadas soportando un sometimiento dictatorial.

${ }^{25}$ Im, Hyug Baeg (2004): «Faltering democratic consolidation in South Korea: democracy at the end of the 'Three Kims' era», Democratization, vol. 11, núm. 5, p. 183. 


\section{LA RELACIÓN ENTRE LA POBREZA, EL DESEMPLEO Y EL CRIMEN ORGANIZADO EN GANGNAM BLUES Y NAMELESS GANGSTER}

Gangnam Blues, ambientada en los comienzos de la década de los 70, se inicia con los planos, desde un helicóptero, de unos campos no urbanizados que acabarán siendo de gran interés para la clase política y las organizaciones criminales del país. Esa zona rural, poco densamente poblada y situada cerca de Seúl, sufrirá las consecuencias de convertirse en el objetivo del próximo bombazo inmobiliario en la nación. Cerca de allí, en otra zona deprimida y poco urbanizada, la película nos muestra a gente pobre que vive en barriadas de chabolas, y que es expulsada de forma violenta del lugar, al ver cómo sus humildes hogares son destruidos con maquinaria pesada acompañada de bandas de matones.

Esa gente no sólo pierde sus hogares sino el poco sustento que tenían -venta de chatarra, recogida de desperdicios, etc.- y se queda sin salida: es en ese momento cuando las bandas criminales están en auge y necesitan más empleados, así que aprovechan la situación de muchos jóvenes sin hogar, sin trabajo, sin sustento ni futuro y los enrolan en sus organizaciones. De este modo vemos cómo una generación de jóvenes abandonados a su suerte por el Estado y la clase política acaban sustentando al crimen organizado, haciendo que la pobreza alimente al crimen.

Los protagonistas de Gangnam Blues, dos jóvenes pobres expulsados de sus recién destruidos hogares, se unen a una banda mafiosa. Inicialmente se muestran reticentes a unirse a la banda, pero reciben ropa nueva y comida, más de lo que recibían de la sociedad y las instituciones públicas, y, a cambio, los protagonistas, junto a otros jóvenes -que probablemente han tenido un pasado similar al de los dos protagonistas-, tienen que reventar el acto de campaña de un partido político.

No obstante, aunque lo que más se muestra en Gangnam Blues es cómo la situación precaria de la juventud surcoreana la llevó a introducirse en bandas criminales en los ańos 70, también este proceso ocurre con personas en una edad más madura. En Nameless Gangster, el protagonista, Choi Ik-hyun, interpretado por Choi Min-sik, un antiguo funcionario del puerto de Busan que acaba convertido en mafioso, le ofrece un puesto de matón en su banda a su cuñado, un sencillo hombre de mediana edad que, pese a tener trabajo, éste no es considerado lo suficientemente digno y bien pagado como para ser el sostén económico de su familia. Es así como se plantea otra situación: la presión a la que están sometidos los hombres en esa sociedad patriarcal surcoreana de los años 80 , que les hace tratar de obtener mayores beneficios económicos para mantener a su familia aun a costa de convertirse en criminales. Se trata, pues, de sociedad intensamente patriarcal ${ }^{26}$ que pone por encima el tener una economía desahogada y poder dar una buena vida a la propia familia a ser un buen ciudadano y cumplir la ley, lo que es paradójico porque

${ }^{26}$ Kim, Nadia Y. (2006): “Patriarchy is So Third World”: Korean Immigrant Women and "Migrating” White Western Masculinity», Social Problems, vol. 53, núm. 4, p. 523. 
es el mismo Estado corrupto que ignora la pobreza y la situación de su población el que empuja al crimen a sus ciudadanos. Es el propio país el que crea un problema que ha de tener que solucionar posteriormente.

De forma similar, en Gangnam Blues un antiguo miembro de un clan mafioso que había dejado su vida criminal atrás por ser poco ética se encuentra en una situación económica desesperada, con muchas deudas y una hija a la que mantener. Así, este antiguo criminal reflexiona sobre la dificultad de reintegrarse socialmente y de vivir dignamente con el poco dinero que un negocio honrado ofrece. ¿No es esa precariedad una forma de presionar a la gente hacia el mundo del crimen? ¿Cómo puede seguirse una vida con ética y dignidad si apenas tienes con qué comer?

Sin embargo, se observa una evolución entre cómo los años 70 y 80 son representados con respecto a los temas de la pobreza, el desempleo y el crimen organizado. Si en los años 70 representados en Gangnam Blues se observa un país con grandes desigualdades, donde sólo existen las dos únicas opciones de la pobreza o el crimen y la corrupción, en la representación que Nameless Gangster ofrece de los años 80 hay una mayor cantidad de matices a este respecto.

En Nameless Gangster los años 80 aparecen representados como un periodo de mayor bonanza económica. De hecho, no es casual que el personaje protagonista escogido fuera un funcionario del puerto de Busan. El puerto de Busan, segunda ciudad más importante de Corea del Sur, experimentó un crecimiento a partir de la década de los 70 , que ayudó precisamente al crecimiento económico y poblacional de la ciudad.

El progreso de Busan, además, funciona en esta película como una alegoría del estado del país en ese momento: cierto optimismo, debido al relajamiento de la dictadura a principios de los ańos 80 , un cierto crecimiento económico y una estabilidad laboral personificada en el protagonista, que es funcionario público. Dicho puerto, además, ha sido uno de los más importantes del mundo en este siglo XXI, siendo el cuarto puerto del mundo en tráfico de contenedores en $2003^{27}$, con lo que Nameless Gangster establece un paralelismo entre la explosión figurada del puerto de Busan y el milagro económico surcoreano desde principios de los años 90.

Existe un elemento crucial de posicionamiento de los autores de ambas películas y que marca la forma en que estas dos obras ejercen su crítica hacia las instituciones: el punto de vista desde el cual están narradas. Gangnam Blues está contada desde el punto de vista de dos jóvenes vagabundos que se convierten en gángsteres. La película juega con el hecho de que, pese a pertenecer a familias mafiosas, estos personajes son expuestos de forma que el espectador pueda empatizar con ellos, oponiéndolos a los personajes políticos, que son mostrados como algo ajeno y negativo. Se transmite así el mensaje de que, mientras los pobres que se han unido a la mafia no tenían muchas más opciones para sobrevivir, los políticos sí y eso los convierte en los verdaderos villanos de la película. En Nameless Gangster, hay una escena en

27 Frémont, A. y Ducruet, C. (2005): «The emergence of a mega-port: From the global to the local, the case of Busan", Tijdschrift voor economische en sociale geografie, vol. 96, núm. 4, p. 421. 
que un joven fiscal encuentra a su mentor, un viejo fiscal, con el personaje protagonista en una cena, en un momento en que este personaje ya es sospechoso de pertenencia a una organización criminal. La película reflexiona sobre el punto de vista del mismo modo que Gangnam Blues: el punto de vista siempre es el de los criminales, lo que hace que fuerza al espectador a empatizar con ellos. Sin embargo, el verdadero héroe pese a sus amorales métodos, el único que no es un corrupto, el joven fiscal, es quien es retratado como alguien despiadado, como alguien ambicioso, alguien sin compasión.

\section{MAFIA, CLASE POLÍTICA Y CORRUPCIÓN EN GANGNAM BLUES Y NAMELESS GANGSTER}

Nameless Gangster y Gangnam Blues se sitúan, respectivamente, en las dos regiones más poderosas del país: Seúl, de la que Gangnam es un distrito ${ }^{28}$, y Busan, segunda ciudad más importante de Corea del Sur y uno de los grandes puertos de Asia, como ya se ha comentado. Estas dos películas funcionan muy bien juntas para exponer cómo, en los dos centros económicos del país, la corrupción reinó desde los años 70, y la mafia y la clase política se aliaron para hacer negocios en los mayores núcleos de poder del Estado.

Gangnam Blues comienza en los años 70. Se muestran unos planos en helicóptero sobre una zona de campo sin urbanizar, que corresponde al distrito de Gangnam, cerca de Seúl, como ya se ha comentado en el epígrafe anterior. Ese repaso visual de la zona sirve para presentar el lugar que iba a ser fruto de especulación inmobiliaria durante los próximos años, y que iba a desembocar en la creación de una de las zonas más exclusivas de Seúl. Tras observar el potencial de la zona, la clase política, en connivencia con algunas organizaciones criminales que les harán el trabajo sucio, planean cómo hacerse con esos terrenos. La película muestra cómo engañan y amenazan a los dueños de las tierras para que las vendan baratas.

La primera secuencia de Nameless Gangster se sitúa en 1990, con la campaña del presidente Roh Tae Woo por acabar con el crimen organizado ${ }^{29}$. Acto seguido, la película muestra al fiscal jefe de Busan amenazando y golpeando durante el interrogatorio al personaje protagonista, que había sido detenido en ese momento por diversos crímenes. La crudeza con la que se narra esta escena es sintomática de dos cuestiones: por un lado, la visión tan directa de uno de los principales miembros de la justicia surcoreana golpeando a un detenido enseña hasta qué punto, incluso con una voluntad de hacer el bien -detener al crimen-, las instituciones públicas

${ }^{28}$ El distrito de Gangnam es, actualmente, uno de los más exclusivos del país. De ahí viene el título de la famosa canción Gangnam Style, del cantante Psy, que hace referencia al lujo y al alto nivel de vida de dicho barrio de Seúl.

${ }_{29}$ Roh Tae-woo fue el primer presidente elegido democráticamente de Corea del Sur. Su mandato duró desde febrero de 1988 hasta febrero de 1993. En 1990 comenzó una cruzada contra la corrupción y el crimen organizado. Irónicamente, en 1997 fue condenado por corrupción. 
han cometido ilegalidades y han tenido comportamientos moralmente cuestionables; por otro lado, esta escena es un gran ejemplo del despertar de la conciencia histórica y social de los cineastas surcoreanos y del papel del cine negro de la ola coreana en ese despertar.

Poco más tarde, cuando la película vuelve al inicio de la historia, en los años 80 , se muestra el germen de la corrupción que se va a apoderar del protagonista: el personaje, funcionario público como ya se ha mencionado anteriormente, es uno de los únicos cuatro empleados encargados de vigilar las mercancías de todo el puerto de Busan. Esto ya implica una velada crítica a las instituciones públicas, que dedicaban pocos esfuerzos a la vigilancia de uno de los principales puntos de exportaciones e importaciones del país. Pero, además, con el poco control existente en el puerto se daban las circunstancias propicias para que los funcionarios se corrompieran: comienzan así, el protagonista y el resto de trabajadores, a cobrar sobornos a cambio de introducir contrabando por el puerto. Más tarde roban mercancías de los contenedores del puerto. Uno de los contenedores inspeccionados por ellos contiene droga, que es abandonada por sus dueños y queda bajo la responsabilidad del protagonista. Éste decide, en lugar de entregarla a las autoridades, robarla e intentar vendérsela a un clan mafioso. Es así como entra en contacto, por primera vez, con el crimen organizado, y es así como, sin que haya una relación directa inicialmente, se muestra la colaboración entre un miembro de las instituciones públicas un funcionario en este caso- y el crimen organizado. Esa relación se va a ir convirtiendo poco a poco en una más estrecha y con mayores implicaciones a medida que avanzan las tramas de la película.

Nameless Gangster también expone cómo, al margen de grandes figuras políticas corruptas, el país funcionaba con pequeñas corruptelas y sobornos. En una escena intrascendente para la narración principal de la historia, pero fundamental para entender el funcionamiento del país que representa la película, se muestra cómo el protagonista consigue arreglar una cuestión burocrática -no se aclara exactamente cuál es esa cuestión concreta, sólo se habla de la obtención de unas licencias- simplemente invitando a comer al grupo de funcionarias administrativas que gestionaban ese trámite. En otra escena similar, el personaje protagonista se jacta de que, con una simple cena cara, uno de los fiscales de la ciudad comenzó a tratarle con respeto. Estas escenas vienen a exponer cómo las instituciones públicas del país de ese momento no sólo eran corruptas sino mediocres, y que los representantes públicos se conformaban con pequeños sobornos para darse por compensados.

En otra escena significativa, el protagonista, detenido junto a sus secuaces por un grupo de policías de bajo rango, los amenaza en la comisaría y los insta a llamar al jefe de policía para demostrarles que se conocen y que no debería estar detenido. Uno de los policías que ha detenido al personaje protagonista llama a su jefe y comprueba, avergonzado, que era cierto que se conocían y acto seguido le libera junto a sus matones.

Otro interesante rasgo de la sociedad surcoreana de la época que se muestra en Nameless Gangster es cómo el parentesco afectaba a las relaciones en el país. Las relaciones de parentesco en Nameless Gangster sirven para estrechar lazos y para establecer jerarquías entre las personas. Así es como el protagonista se hace amigo 
en 1985 de un fiscal que luego va a ayudar a liberarle en 1990 cuando sea detenido y acusado por el joven fiscal. En la escena de 1985, cuando el protagonista y el viejo fiscal se conocen, se muestra cómo el protagonista se acerca a él a través de un supuesto parentesco existente entre ambos.

De hecho, el peso de las relaciones familiares y los parentescos va a tener también importancia en cómo el protagonista acabará estrechando su relación con el jefe de la banda mafiosa a la que le vende la droga al principio de la película. En ese sentido, las arcaicas jerarquías familiares son también una representación de aquella época en el país. Es decir, ese arcaísmo familiar llegaba a un extremo ridículo, tal y como lo muestra la película: que un joven pero fuerte líder mafioso tuviera que acabar rindiendo pleitesía a un funcionario don nadie sólo por familiar suyo ${ }^{30}$ y por ser mayor que él en edad.

En una secuencia que puede ser interpretada con gran ironía, el jefe de la banda criminal pronuncia la siguiente frase: «Padrino, el estudiante lo es si estudia. El gángster lo es si lucha». Así, este personaje critica las formas de actuar de su padrino (de nuevo, el tema del parentesco), el protagonista, que ha pasado de luchar contra bandas rivales a tratar de ganar influencia a través de contactos políticos y tratos empresariales. Éste parece, en cierto modo, un punto de inflexión, ya que refleja la transformación de la banda mafiosa y del país: la organización criminal deja sus negocios sucios a un lado y se infiltra en el mismo Estado para hacer crecer a la organización. Esa infiltración llega a los mismos partidos políticos puesto que Nameless Gangster muestra cómo miembros del clan mafioso se introducen en las juventudes de uno de los partidos.

El final de Nameless Gangster construye una representación agridulce de la Corea del Sur contemporánea. El protagonista, acorralado por las acusaciones del joven fiscal, decide traicionar a los miembros de su organización mafiosa para evitar ser juzgado por delitos graves, lo que le lleva a una jubilación tranquila alejada del mundo del crimen. No obstante, la película finaliza con un mensaje ambiguo: uno de los hijos del protagonista se hace jurista y comienza a ejercer en la fiscalía, pero de forma ética. Se trata, por tanto, de una evolución: los hijos de los criminales que se enriquecieron en los años 80 gracias a la corrupción y al tráfico de drogas más tarde usarían ese dinero e influencia para labrarse una carrera honorable y obtener una buena reputación en sus profesiones.

${ }^{30}$ En la película sucede repetidamente que dos personajes se conocen y acaban dándose cuenta de que provienen del mismo tronco familiar. Da una idea de las intrincadas relaciones familiares existentes en todo el país en la época. 


\section{CONCLUSIONES}

Pese a parecer que la ola coreana podría ser un fenómeno complaciente institucionalmente y apolítico por su enorme fuerza comercial e industrial, películas como Nameless Gangster y Gangnam Blues demuestran que se trata de un movimiento, al menos en lo cinematográfico, con conciencia social y capacidad de reflexión histórica.

Estas dos películas de temática mafiosa se sirven de las herramientas del género negro para exponer diversas cuestiones problemáticas disfrazadas de cine de entretenimiento. Así, empleando la narrativa y el look de los thrillers criminales, Nameless Gangster y Gangnam Blues exploran el debate de cómo la actualidad de Corea del Sur está fuertemente configurada por las convulsas décadas de los $70 \mathrm{y}$ 80 , unos años en que la corrupción política y el crimen organizado eran una de las principales preocupaciones de la ciudadanía, al margen del autoritarismo del Estado.

Ambas obras, además, se centran en el papel de los actores políticos e institucionales en la proliferación del crimen organizado, dejando en un segundo plano ciertos tópicos de este tipo de cine, como el tráfico de drogas o el enfrentamiento entre bandas criminales. Se trata, por tanto, de una osada apuesta que huye de la retroalimentación de los códigos del propio género y decide emprender un camino menos transitado y más socialmente contestatario. 